Nervenarzt 2016 $\cdot 87: 803-804$

DOI 10.1007/s00115-016-0165-2

Online publiziert: 29. Juni 2016

๑) Springer-Verlag Berlin Heidelberg 2016

CrossMark

Liebe Kolleginnen, liebe Kollegen,

vom 21. bis 24. September treffen sich in Mannheim voraussichtlich mehr als 6000 Neurologen und Neurowissenschaftler zum 89. Kongress der Deutschen Gesellschaft für Neurologie (DGN) mit Fortbildungsakademie. Wir laden Sie herzlich dazu ein! Die Veranstaltung, die wir als Kongresspräsident und Kongresssekretär aus der Neurologischen Klinik der Friedrich-Alexander-Universität Erlangen-Nürnberg in diesem Jahr organisieren, ist das zentrale Wissenschafts-, Fortbildungs- und Diskussionsforum der neurologischen Medizin und einer der größten Medizinkongresse Deutschlands. Die Besucher erwartet ein hochkarätiges Programm, das in vier Tagen das gesamte Spektrum der Neurologie abdeckt: mit 80 wissenschaftlichen Sessions und mehr als 580 wissenschaftlichen Vorträgen, 315 Postern, 23 Industriesymposien, einer großen Fachausstellung sowie dem berufspolitischen Programm im DGN(forum und dem Nachwuchsprogramm. Ferner werden 85 Aussteller und 20 Vereine und Verbände in Mannheim vertreten sein. Hinzu kommt das unabhängige Programm der DGN-Fortbildungsakademie mit 55 Fortbildungskursen und insgesamt 320 Vorträgen, in dem praxisnahe Themen von den besten Referenten der deutschen Neurologie präsentiert werden.

\section{) Die Neurologie ist heute eine therapeutisch hoch aktive Disziplin}

Einige Highlights und Themen aus dem wissenschaftlichen Programm des dies-

\author{
S. Schwab · H. B. Huttner \\ Neurologischen Klinik, Universitätsklinikum Erlangen, Erlangen, Deutschland
}

\title{
Invasive Neurologie im Fokus
}

jährigen DGN-Kongresses werden in der vorliegenden Ausgabe von Der Nervenarzt exemplarisch dargestellt. Wir haben als Titel des diesjährigen Präsidentensymposiums das Thema „Invasive Neurologie“ gewählt. Als Leser könnten Sie der Meinung sein, dass es sich hier um eine Kontradiktion handelt - aber das Gegenteil ist der Fall! Hat sich doch die Neurologie, die jahrelang als eher diagnostisches Fach wahrgenommen wurde, innerhalb der letzten 20 Jahre zu einer therapeutisch hoch aktiven Disziplin gewandelt, in der mehr und mehr auch invasive Therapie angeboten werden. Dieser Entwicklung wollen wir im Präsidentensymposium Rechnung tragen und anhand der klinischen Schwerpunkte der Neurologischen Universitätsklinik in Erlangen diskutieren.

Zunächst werden F. Marxreiter, A. Storch und J. Winkler einen Überblick über „Zellersatz und adulte Neurogenese beim idiopathischen ParkinsonSyndrom" geben. Gerade die Zellersatztherapie mit der Transplantation neuraler (Vorläufer-)Zellen ist ein hoch spannendes und sehr dynamisches Feld, das momentan schon den Schritt in klinische Prüfstudien nimmt. In den vergangenen Jahren haben sich auch in der Neuroimmunologie immer mehr neue invasivere und aggressive Therapieoptionen ergeben. M. Diebold, L. Kappos und T. Derfuss werden in ihrem Beitrag "Zelldepletion und Myeloablation bei neuroimmunologischen Erkrankungen “ die Therapieoptionen und Gefahren von Stammzelltransplantationen und selektiven monoklonalen Antikörpern bei der Multiplen Sklerose darlegen. Einen Quantensprung hat es in der Schlaganfalltherapie seit letztem Jahr durch die positiven Thrombektomiestudien gege- ben. L. Breuer und M. Köhrmann gehen in ihrem Beitrag „Invasive Schlaganfallbehandlung - Lost in Translation?" der Frage nach, wie sich die positiven Ergebnisse der Thrombektomiestudien in die klinische Routine übertragen lassen und beleuchten Themen wie regionale Aspekte, Zentralisierung der Versorgung sowie Implementierung der Intervention im klinischen Alltag. Die invasive Ableitung und Stimulationsverfahren gewinnen in der Epileptologie in den letzten Jahren immens an Bedeutung. A. Schulze-Bonhage, H. Hamer, M. Hirsch und M. Hagge gehen in ihrem Beitrag ,Invasive Stimulationsverfahren und EEG-Diagnostik bei Epilepsien" auf Verfahren wie Vagusstimulation ein und geben einen Überblick zu den Möglichkeiten von Tiefenelektroden im Rahmen der prächirurgischen Diagnostik bei der Versorgung fokaler Epilepsien. Abschließend - sozusagen als Ergänzung zu invasiven Diagnostik- und Therapiekonzepten - beschreiben $D$. Antonenko und A. Flöel in ihrem Artikel „Nichtinvasive Stimulationsverfahren in der Neurologie" den Stellenwert der transkraniellen Gleichstromstimulation als wertvolles Therapieverfahren zur Behandlung degenerativer oder neurovaskulärer Erkrankungen.

Sie werden durch die Beiträge erkennen, dass in nahezu allen Bereichen der Neurologie die Invasivität in Diagnostik und Therapie in den letzten Jahren zugenommen hat. Wichtig und ermutigend ist, dass der positive Einfluss auf das funktionelle Langzeitergebnis von Patienten so zunehmend mit Evidenz untermauert wird. Weitere spannende Ergebnisse sind auf dem Kongress zu erwarten und zu diskutieren. Wir freuen uns auf einen anregenden Wissens- und Erfahrungsaustausch in Mannheim! 


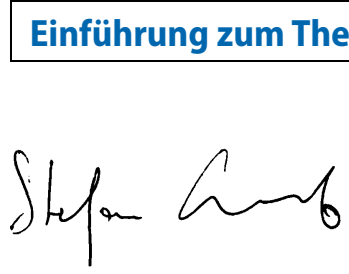

Prof. Dr. Stefan Schwab

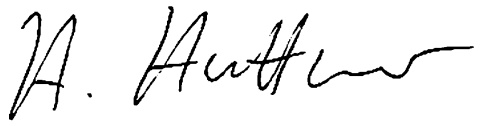

Prof. Dr. Hagen B. Huttner

\section{Korrespondenzadresse}

\section{Prof. Dr. S. Schwab}

Neurologischen Klinik, Universitätsklinikum Erlangen

Schwabachanlage 6, 91054 Erlangen,

Deutschland

stefan.schwab@uk-erlangen.de

Interessenkonflikt. S. Schwab gibt an, Vortragshonorare der Firmen Bayer, BMS Pfizer und Boehringer Ingelheim erhalten zu haben. H. B. Huttner gibt an, dass kein Interessenkonflikt besteht.

\section{Leitthemenübersicht}

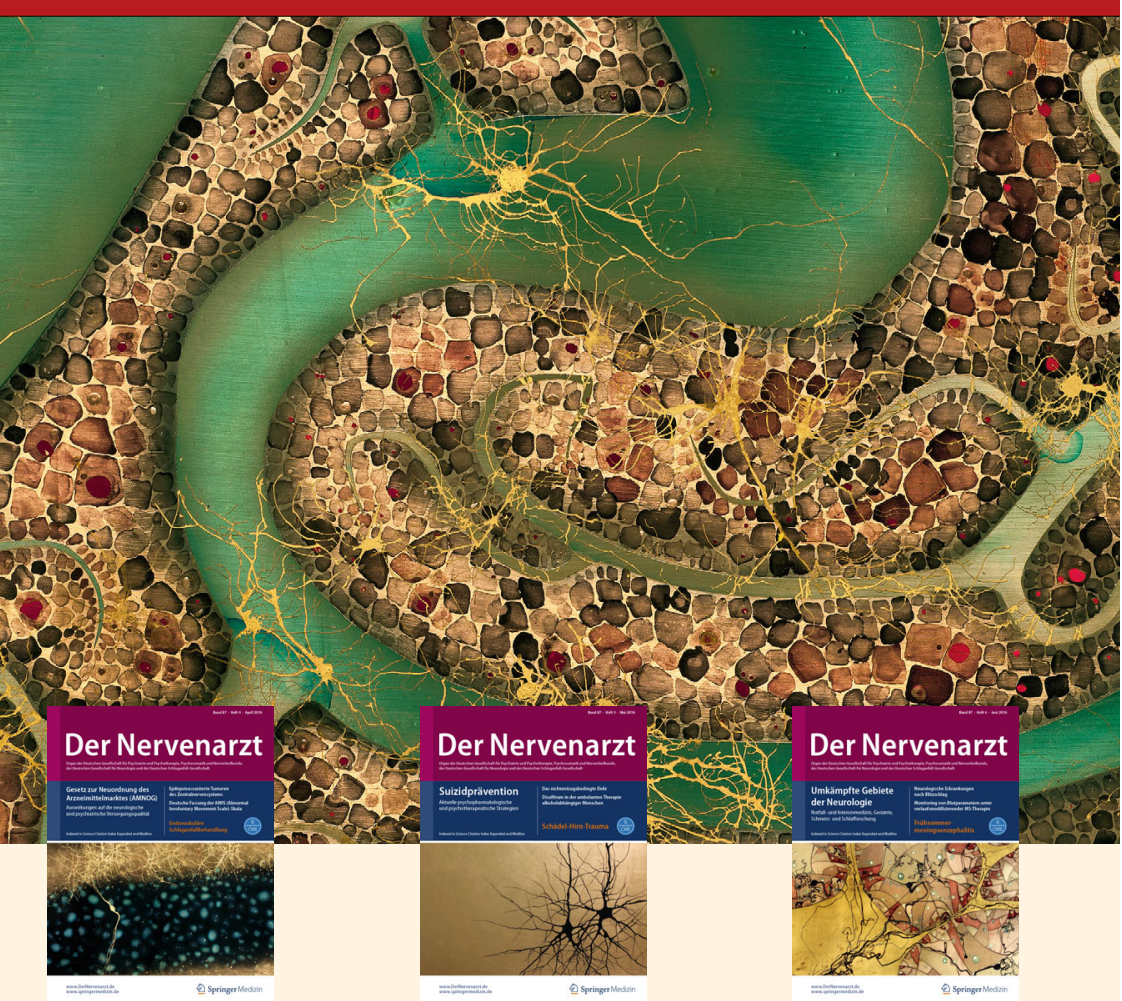

Die Zeitschrift Der Nervenarzt bietet Ihnen jeden Monat umfassende und aktuelle Beiträge zu interessanten Themenschwerpunkten aus allen Bereichen der Neurologie, Psychiatrie, Psychotherapie, Psychosomatik und Nervenheilkunde.

\section{Überblick}

2016

01/16 Störungen durch Alkohol- und Tabakkonsum

02/16 Hirntoddiagnostik

03/16 Intensivmedizin

04/16 Gesetz zur Neuordnung des Arzneimittelmarktes (AMNOG)

05/16 Suizidprävention

06/16 Umkämpfte Gebiete der Neurologie

07/16 kein Leitthema

08/16 Invasive Neurologie im Fokus

09/16 Psychiatrische und neurologische Erkrankungen in Schwangerschaft und Stillzeit

10/16 Evidenzbasierte Neurorehabilitation

11/16 Kongressheft DGPPN 2016

12/16 Liquordiagnostik

\section{Vorschau}

\section{7}

01/17 Psychosoziale Versorgung bei Flüchtlingen

02/17 Telemedizin

03/17 Risikogene bei psychiatrischen und neurologischen Erkrankungen

04/17 Parkinsonsyndrome

05/17 Sexualität und Psychiatrie

06/17 Notfallambulanz

07/17 Sexualmedizin in der Psychiatrie

08/17 Kongressheft DGN 2017

09/17 Kongressheft DGPPN 2017

10/17 Differentialdiagnose epileptischer Anfälle

11/17 Psychosoziale Betreuung bei demenziell Erkrankten und ihren Angehörigen

12/17 Neuropädiatrie
Alle Inhalte von Der Nervenarzt finden Sie unter www.springermedizin.de/der-nervenarzt

SpringerMedizin.de bietet Ihnen Zugang zu allen elektronisch verfügbaren Ausgaben Ihrer Zeitschrift - unabhängig davon, seit wann Sie die Zeitschrift abonniert haben.

Unser Tipp: Mit dem e.Med Kombi-Abo können Sie jederzeit auf alle Inhalte von SpringerMedizin.de zugreifen. Auf Wunsch erhalten Sie darüber hinaus eine gedruckte Fachzeitschrift Ihrer Wahl.

Testen Sie e.Med 30 Tage lang kostenlos und unverbindlich!

Jetzt informieren unter www.springermedizin.de/eMed

oder telefonisch unter 0800-77 80777 (Montag bis Freitag, 10 bis 17 Uhr) 